\title{
Positive Periodic Solution for a Two-Species Predator-Prey System
}

\author{
Meiyu Cao, Xiaoping Li*, Xiangjun Dai \\ Science College, Hunan Agricultural University, Changsha, China \\ Email: ${ }^{\text {Ixpiii168@aliyun.com }}$
}

Received 1 March 2014; revised 1 April 2014; accepted 8 April 2014

Copyright (C) 2014 by authors and Scientific Research Publishing Inc.

This work is licensed under the Creative Commons Attribution International License (CC BY).

http://creativecommons.org/licenses/by/4.0/

(c) (i) Open Access

\section{Abstract}

A two-species predator-prey system with time delay in a two-patch environment is investigated. By using a continuation theorem based on coincidence degree theory, we obtain some sufficient conditions for the existence of periodic solution for the system.

\section{Keywords}

Predator-Prey System, Diffusion, Periodic Solution, Coincidence Degree

\section{Introduction}

Dynamical systems generated by predator-prey models have long been the topic of research interest of many biomathematical scholars, and there have been vast studies to investigate the dynamics of predator-prey models, see e.g., Refs. [1]-[12] and references therein. In 1975, Beddington [13] and DeAngelis [14] proposed the predator-prey system with the Beddington-DeAngelis functional response as follows.

$$
\left\{\begin{array}{l}
x^{\prime}=x\left(a-b x-\frac{c y}{m_{1}+m_{2} x+m_{3} y}\right), \\
y^{\prime}=y\left(-d+\frac{f x}{m_{1}+m_{2} x+m_{3} y}\right),
\end{array}\right.
$$

In the last years, some experts have studied the system [15]-[21]. Recently, Li and Takeuchi [22] proposed the following model with both Beddington-DeAngelis functional response and density dependent predator

*Corresponding author. 


$$
\left\{\begin{array}{l}
x^{\prime}=x\left(a-b x-\frac{c y}{m_{1}+m_{2} x+m_{3} y}\right), \\
y^{\prime}=y\left(-d-e y+\frac{f x}{m_{1}+m_{2} x+m_{3} y}\right),
\end{array}\right.
$$

and discussed the dynamic behaviors of the model. In this paper, we consider the following nonautonomous two-species predator-prey system with diffusion and time delays.

$$
\left\{\begin{array}{l}
x_{1}^{\prime}(t)=x_{1}(t)\left[a_{1}(t)-b_{1}(t) x_{1}(t)-h_{1}(t)-\frac{c(t) x_{3}(t)}{m_{1}(t)+m_{2}(t) x_{1}(t)+m_{3}(t) x_{3}(t)}\right]+D_{1}(t)\left[x_{2}(t)-x_{1}(t)\right] \\
x_{2}^{\prime}(t)=x_{2}(t)\left[a_{2}(t)-b_{2}(t) x_{2}(t)\right]+D_{2}(t)\left[x_{1}(t)-x_{2}(t)\right] \\
x_{3}^{\prime}(t)=x_{3}(t)\left[-d(t)-e(t) x_{3}(t)-h_{2}(t)-\frac{f(t) x_{1}(t-\tau)}{m_{1}(t)+m_{2}(t) x_{1}(t-\tau)+m_{3}(t) x_{3}(t-\tau)}\right]
\end{array}\right.
$$

where $x_{i}(t)$ represents the prey population in the ith patch $(i=1,2)$, and $x_{3}(t)$ represents the predator population. $D_{i}(t)$ denotes the dispersal rate of the prey in the ith patch $(i=1,2)$. We always make the following fundamental assumptions for system (1.3): $\tau$ is positive constant and $a_{i}(t)(i=1,2), b_{i}(t), h_{i}(t), D_{i}(t)$, $c(t), d(t), e(t), f(t), m_{1}(t), m_{2}(t), m_{3}(t)$ re positive continuous $\omega$-periodic functions.

The main purpose of this paper is, by using the coincidence degree theory to derive the sufficient conditions for the existence of periodic solution of (1.3).

\section{Preliminaries}

The method to be used in this paper involves the applications of the continuation theorem of coincidence degree. we shall use some concepts and results from the book by Gaines and Mawhin [23].

Let $X, Z$ be real Banach spaces, $L: D o m L \subset X \rightarrow Z$ be a linear mapping, and $N: X \rightarrow Z$ be a continuous mapping. The mapping $L$ is called a Fredholm mapping of index zero if

$$
\operatorname{dim} \operatorname{Ker} L=\operatorname{codim} \operatorname{Im} L<+\infty
$$

and $\operatorname{Im} L$ is closed in $Z$. If $L$ is a Fredholm mapping of index zero and there exist continuous projectors $P: X \rightarrow X$ and $Q: Z \rightarrow Z$ such that $\operatorname{Im} P=\operatorname{Ker} L, \operatorname{Im} L=\operatorname{Ker} Q=\operatorname{Im}(I-Q)$, then the restriction $L_{P}$ of $L$ to $\operatorname{Dom} L \cap \operatorname{Ker} P:(I-P) X \rightarrow \operatorname{Im} L$ is invertible. Denote the inverse of $L_{P}$ by $K_{P}$. If $\Omega$ is an open bounded subset of $X$, the mapping $N$ will be called $L$-compact on $\bar{\Omega}$ if $Q N(\bar{\Omega})$ is bounded and $K_{P}(I-Q) N: \bar{\Omega} \rightarrow X$ is compact. Since $\operatorname{Im} Q$ is isomorphic to $\operatorname{KerL}$, there exists isomorphism $J: \operatorname{Im} Q \rightarrow \operatorname{Ker} L$.

Lemma 2.1 (Continuation theorem [23]) Let $\Omega \subset X$ be an open bounded set, $L$ be a Fredholm mapping of index zero and $N$ be $L$-compact on $\bar{\Omega}$. Assume

1) for each $\lambda \in(0,1), \quad x \in \partial \Omega \cap D o m L, \quad L x \neq \lambda N x$;

2) for each $x \in \operatorname{Ker} L \cap \partial \Omega, Q N x \neq 0$;

3) $\operatorname{deg}\{J Q N, \Omega \cap \operatorname{KerL}, 0\} \neq 0$.

Then $L x=N x$ has at least one solution in $D o m L \cap \bar{\Omega}$.

Throughout this paper, we adopt the notations $\bar{f}=\frac{1}{\omega} \int_{0}^{\omega} f(t) \mathrm{d} t, \quad f^{l}=\min _{t \in[0, \omega]}|f(x)|, \quad f^{M}=\max _{t \in[0, \omega]}|f(x)|$, where $f(t)$ is an $\omega$-periodic continuous function.

\section{Main Result}

Theorem 3.1 Assume that

1) $a_{1}(t)-h_{1}(t)-D_{1}(t)>0$

2) $h_{2}(t)-D_{2}(t)>0$; 


$$
\begin{aligned}
& \text { 3) } \overline{a_{1}(t)-h_{1}(t)-D_{1}(t)}-\overline{\left(\frac{c}{m_{3}}\right)}>0 \text {; } \\
& \text { 4) } \overline{\left(\frac{f}{m_{2}}\right)}-\overline{d+h_{2}}>0 \text {. }
\end{aligned}
$$

Then system (1.3) has at least one positive $\omega$-periodic solution.

Proof. Let $x_{i}(t)=\mathrm{e}^{u_{i}(t)}, \quad u_{i}^{\prime}(t)=Y_{i}(t)(i=1,2,3), \quad u=\left(u_{1}, u_{2}, u_{3}\right)^{\mathrm{T}}, \quad u^{\prime}=\left(u_{1}^{\prime}, u_{2}^{\prime}, u_{3}^{\prime}\right)^{\mathrm{T}}, \quad Y=\left(Y_{1}, Y_{2}, Y_{3}\right)^{\mathrm{T}}$, then (1.3) can be rewritten as follows:

$$
\left\{\begin{array}{l}
Y_{1}(t)=a_{1}(t)-h_{1}(t)-b_{1}(t) \mathrm{e}^{u_{1}(t)}-\frac{c(t) \mathrm{e}^{u_{3}(t)}}{m_{1}(t)+m_{2}(t) \mathrm{e}^{u_{1}(t)}+m_{3}(t) \mathrm{e}^{u_{3}(t)}}+D_{1}(t) \mathrm{e}^{u_{2}(t)-u_{1}(t)} \\
Y_{2}(t)=a_{2}(t)-b_{2}(t) \mathrm{e}^{u_{2}(t)}+D_{2}(t) \mathrm{e}^{u_{1}(t)-u_{2}(t)} \\
Y_{3}(t)=-d(t)-h_{2}(t)-e(t) \mathrm{e}^{u_{3}(t)}-\frac{f(t) \mathrm{e}^{u_{1}(t-\tau)}}{m_{1}(t)+m_{2}(t) \mathrm{e}^{u_{1}(t-\tau)}+m_{3}(t) \mathrm{e}^{u_{3}(t-\tau)}}
\end{array}\right.
$$

where all function are defined as ones in system (1.3). It is easy to know that if (3.1) has one $\omega$-periodic solution $\left(x_{1}^{*}(t), x_{2}^{*}(t), x_{3}^{*}(t)\right)^{\mathrm{T}}$, then $\left(\mathrm{e}^{x_{1}^{*}(t)}, \mathrm{e}^{x_{2}^{*}(t)}, \mathrm{e}^{x_{3}^{*}(t)}\right)^{\mathrm{T}}$ is a positive $\omega$-periodic solution of system (1.3) Therefore, to complete the proof , it suffices to show that system (3.1) has one $\omega$-periodic solution.

Take $X=Z=\left\{u \in C\left(R, R^{3}\right): u(t+\omega)=u(t)\right\}$ and $\|u\|=\sum_{i=1}^{3} \max _{t \in(0, \omega)}\left|u_{i}(t)\right|$, then $X$ and $Z$ are Banach space with the norm $\|\cdot\|$.

Set $L u=u^{\prime}, \quad N u=Y, P u=\frac{1}{\omega} \int_{0}^{\omega} u(t) \mathrm{d} t, Q u=\frac{1}{\omega} \int_{0}^{\omega} u(t) \mathrm{d} t, \quad u \in X$. Obviously, $\operatorname{Ker} L=R^{3}$, $\operatorname{Im} L=\left\{u \in X: \int_{0}^{\omega} u(t) \mathrm{d} t=0\right\}$ is closed in $Z$ and $\operatorname{dim} \operatorname{Ker} L=\operatorname{codim} \operatorname{Im} L=3$. Therefore, $L$ is a Fredholm mapping of index zero. Through an easy computation we find that the inverse $K_{p}$ of $L_{p}$ has the form $K_{p}: \operatorname{Im} L \rightarrow \operatorname{Dom} L \bigcap \operatorname{Ker} P, \quad K_{p}(u)=\int_{0}^{\omega} u(s) \mathrm{d} s-\frac{1}{\omega} \int_{0}^{\omega} \int_{0}^{\eta} u(t) \mathrm{d} t \mathrm{~d} \eta$. Clearly, $Q N$ and $K_{P}(I-Q) N$ are continuous. By using Arzela-Ascoli theorem, it is not difficult to prove that $\overline{K_{P}(I-Q) N(\bar{\Omega})}$ is compact for any open bounded set $\Omega \in X$. Moreover, $Q N(\bar{\Omega})$ is bounded. Therefore, $N$ is $L$-compact on $\bar{\Omega}$ with any open bounded set $\Omega \in X$.

Corresponding to the operator equation $L u=\lambda N u, \lambda \in(0,1)$, we have

$$
u^{\prime}(t)=\lambda Y(t)
$$

Suppose that $u \in X$ is a solution of (3.2) for an appropriate $\lambda \in(0,1)$. Integrating (3.2) over the interval $[0, \omega]$ leads to

$$
\begin{gathered}
\int_{0}^{\omega}\left(a_{1}(t)-h_{1}(t)-D_{1}(t)\right) \mathrm{d} t+\int_{0}^{\omega} D_{1}(t) \mathrm{e}^{u_{2}(t)-u_{1}(t)} \mathrm{d} t \\
=\int_{0}^{\omega} b_{1}(t) \mathrm{e}^{u_{1}(t)} \mathrm{d} t+\int_{0}^{\omega} \frac{c(t) \mathrm{e}^{u_{3}(t)}}{m_{1}(t)+m_{2}(t) \mathrm{e}^{u_{1}(t)}+m_{3}(t) \mathrm{e}^{u_{3}(t)}} \mathrm{d} t, \\
\int_{0}^{\omega}\left(a_{2}(t)-D_{2}(t)\right) \mathrm{d} t+\int_{0}^{\omega} D_{2}(t) \mathrm{e}^{u_{1}(t)-u_{2}(t)} \mathrm{d} t=\int_{0}^{\omega} b_{2}(t) \mathrm{e}^{u_{2}(t)} \mathrm{d} t, \\
\int_{0}^{\omega}\left(d(t)+h_{2}(t)\right) \mathrm{d} t+\int_{0}^{\omega} e(t) \mathrm{e}^{u_{3} t} \mathrm{~d} t=\int_{0}^{\omega} \frac{f(t) \mathrm{e}^{u_{1}(t-\tau)}}{m_{1}(t)+m_{2}(t) \mathrm{e}^{u_{1}(t-\tau)}+m_{3}(t) \mathrm{e}^{u_{3}(t-\tau)}} \mathrm{d} t .
\end{gathered}
$$

From (3.2)-(3.5), we have 


$$
\begin{aligned}
& \int_{0}^{\omega}\left|u_{1}^{\prime}(t)\right| \mathrm{d} t \leq \int_{0}^{\omega}\left(a_{1}(t)-h_{1}(t)-D_{1}(t)\right) \mathrm{d} t+\int_{0}^{\omega} b_{1}(t) \mathrm{e}^{u_{1}(t)} \mathrm{d} t \\
& +\int_{0}^{\omega} \frac{c(t) \mathrm{e}^{u_{3}(t)}}{m_{1}(t)+m_{2}(t) \mathrm{e}^{u_{1}(t)}+m_{3}(t) \mathrm{e}^{u_{3}(t)}} \mathrm{d} t+\int_{0}^{\omega} D_{1}(t) \mathrm{e}^{u_{2}(t)-u_{1}(t)} \mathrm{d} t \\
& =2 \int_{0}^{\omega} b_{1}(t) \mathrm{e}^{u_{1}(t)} \mathrm{d} t+2 \int_{0}^{\omega} \frac{c(t) \mathrm{e}^{u_{3}(t)}}{m_{1}(t)+m_{2}(t) \mathrm{e}^{u_{1}(t)}+m_{3}(t) \mathrm{e}^{u_{3}(t)}} \mathrm{d} t \\
& <2 b_{1}^{M} \int_{0}^{\omega} \mathrm{e}^{u_{1}(t)} \mathrm{d} t+2 \omega \overline{\left(\frac{c}{m_{3}}\right)}, \\
& \int_{0}^{\omega}\left|u_{2}^{\prime}(t)\right| \mathrm{d} t \leq \int_{0}^{\omega}\left(a_{2}(t)-D_{2}(t)\right) \mathrm{d} t+\int_{0}^{\omega} b_{2}(t) \mathrm{e}^{u_{2}(t)} \mathrm{d} t \\
& =2 \int_{0}^{\omega} b_{2}(t) \mathrm{e}^{u_{2}(t)} \mathrm{d} t<2 b_{2}^{M} \int_{0}^{\omega} \mathrm{e}^{u_{2}(t)} \mathrm{d} t, \\
& \int_{0}^{\infty}\left|u_{3}^{\prime}(t)\right| \mathrm{d} t \leq \int_{0}^{\omega}\left(d(t)+h_{2}(t)\right) \mathrm{d} t+\int_{0}^{\omega} e(t) \mathrm{e}^{u_{3}(t)} \mathrm{d} t \\
& +\int_{0}^{\omega} \frac{f(t) \mathrm{e}^{u_{1}(t-\tau)}}{m_{1}(t)+m_{2}(t) \mathrm{e}^{u_{1}(t-\tau)}+m_{3}(t) \mathrm{e}^{u_{3}(t-\tau)}} \mathrm{d} t \\
& =2 \int_{0}^{\infty} \frac{f(t) \mathrm{e}^{u_{1}(t-\tau)}}{m_{1}(t)+m_{2}(t) \mathrm{e}^{u_{1}(t-\tau)}+m_{3}(t) \mathrm{e}^{u_{3}(t-\tau)}} \mathrm{d} t \\
& <2 \omega \overline{\left(\frac{c}{m_{2}}\right)}=d_{3} \text {. }
\end{aligned}
$$

Multiplying the first equation of (3.2) by $\mathrm{e}^{u_{1}(t)}$ and integrating over $[0, \omega]$ gives.

$$
\int_{0}^{\infty} b_{1}(t) \mathrm{e}^{2 u_{1}(t)} \mathrm{d} t<\int_{0}^{\infty}\left(a_{1}(t)-h_{1}(t)-D_{1}(t)\right) \mathrm{e}^{u_{1}(t)} \mathrm{d} t+\int_{0}^{\omega} D_{1}(t) \mathrm{e}^{u_{2}(t)} \mathrm{d} t,
$$

which implies

$$
b_{1}^{l} \int_{0}^{\omega} \mathrm{e}^{2 u_{1}(t)} \mathrm{d} t<\left(a_{1}-h_{1}-D_{1}\right)^{M} \int_{0}^{\omega} \mathrm{e}^{u_{1}(t)} \mathrm{d} t+D_{1}^{M} \int_{0}^{\omega} \mathrm{e}^{u_{2}(t)} \mathrm{d} t
$$

By using the inequalities

$$
\left(\int_{0}^{\omega} \mathrm{e}^{u_{1}(t)} \mathrm{d} t\right)^{2} \leq \omega \int_{0}^{\omega} \mathrm{e}^{2 u_{2}(t)} \mathrm{d} t
$$

It follows from (3.9) that

$$
\frac{b_{1}^{l}}{\omega}\left(\int_{0}^{\omega} \mathrm{e}^{u_{1}(t)} \mathrm{d} t\right)^{2}<\left(a_{1}-h_{1}-D_{1}\right)^{M} \int_{0}^{\omega} \mathrm{e}^{u_{1}(t)} \mathrm{d} t+D_{1}^{M} \int_{0}^{\omega} \mathrm{e}^{u_{2}(t)} \mathrm{d} t,
$$

This yields

$$
\frac{2 b_{1}^{l}}{\omega} \int_{0}^{\omega} \mathrm{e}^{u_{1}(t)} \mathrm{d} t<\left(a_{1}-h_{1}-D_{1}\right)^{M}+\left\{\left[\left(a_{1}-h_{1}-D_{1}\right)^{M}\right]^{2}+\frac{4 b_{1}^{l} D_{1}^{M}}{\omega} \int_{0}^{\omega} \mathrm{e}^{u_{2}(t)} \mathrm{d} t\right\}^{\frac{1}{2}} .
$$

By using the inequalities

$$
(a+b)^{\frac{1}{2}}<a^{\frac{1}{2}}+b^{\frac{1}{2}}, \quad a>0, b>0 .
$$

It follows from (3.10) that

$$
\frac{b_{1}^{l}}{\omega} \int_{0}^{\omega} \mathrm{e}^{u_{1}(t)} \mathrm{d} t<\left(a_{1}-h_{1}-D_{1}\right)^{M}+\sqrt{\frac{b_{1}^{l} D_{1}^{M}}{\omega}}\left(\int_{0}^{\omega} \mathrm{e}^{u_{2}(t)} \mathrm{d} t\right)^{\frac{1}{2}}
$$


Multiplying the second equation of (3.2) by $\mathrm{e}^{u_{2}(t)}$ and integrating over $[0, \omega]$, similarly, we can obtain

$$
\frac{b_{2}^{l}}{\omega} \int_{0}^{\omega} \mathrm{e}^{u_{2}(t)} \mathrm{d} t<\left(a_{2}-D_{2}\right)^{M}+\sqrt{\frac{b_{2}^{l} D_{2}^{M}}{\omega}}\left(\int_{0}^{\omega} \mathrm{e}^{u_{1}(t)} \mathrm{d} t\right)^{\frac{1}{2}} .
$$

Substitute (3.13) to (3.12), which leads to

$$
\sqrt{b_{2}^{l}} b_{1}^{l} \int_{0}^{\omega} \mathrm{e}^{u_{1}(t)} \mathrm{d} t<\omega \sqrt{b_{2}^{l}}\left(a_{1}-h_{1}-D_{1}\right)^{M}+\sqrt{\omega b_{1}^{l} b_{2}^{l} D_{1}^{M}}\left[\sqrt{\omega\left(a_{2}-D_{2}\right)^{M}}+\sqrt[4]{\omega b_{2}^{l} D_{2}^{M} \int_{0}^{\omega} \mathrm{e}^{u_{1}(t)} \mathrm{d} t}\right]
$$

So, there exist a positive constant $\rho_{1}$ such that

$$
\int_{0}^{\omega} \mathrm{e}^{u_{1}(t)} \mathrm{d} t<\rho_{1} .
$$

It follows from (3.13) and (3.14) that there exist a positive constant $\rho_{2}$ such that

$$
\int_{0}^{\omega} \mathrm{e}^{u_{2}(t)} \mathrm{d} t<\rho_{2} .
$$

Substitute (3.14), (3.15) to (3.6) and (3.7), which leads to

$$
\begin{gathered}
\int_{0}^{\omega}\left|u_{1}^{\prime}\right| \mathrm{d} t<2 b_{1}^{M}+2 \omega \overline{\left(\frac{c}{m_{3}}\right)}=d_{1}, \\
\int_{0}^{\omega}\left|u_{2}^{\prime}\right| \mathrm{d} t<2 b_{2}^{M} \rho_{2}=d_{1} .
\end{gathered}
$$

From (3.3) we have

$$
\begin{aligned}
b_{1}^{M} \int_{0}^{\omega} \mathrm{e}^{u_{1}(t)} \mathrm{d} t & >\int_{0}^{\omega} b_{1}(t) \mathrm{e}^{u_{1}(t)} \mathrm{d} t>\omega\left(\overline{a_{1}-h_{1}-D_{1}}\right)-\int_{0}^{\omega} \frac{c(t) \mathrm{e}^{u_{3}(t)}}{m_{1}(t)+m_{2}(t) \mathrm{e}^{u_{1}(t)}+m_{3}(t) \mathrm{e}^{u_{3}(t)}} \mathrm{d} t \\
& >\omega\left(\overline{a_{1}-h_{1}-D_{1}}\right)-\omega\left(\overline{\left.\frac{c}{m_{3}}\right)}\right.
\end{aligned}
$$

From (3.4) we have

$$
b_{2}^{M} \int_{0}^{\omega} \mathrm{e}^{u_{2}(t)} \mathrm{d} t>\omega \overline{\left(a_{2}-D_{2}\right)} .
$$

It follows from (3.14), (3.15), (3.18) and (3.19) that there exist $t_{i}, t_{i}^{*}(i=1,2)$ such that

$$
\begin{gathered}
\mathrm{e}^{u_{i}\left(t_{i}\right)<\frac{\rho_{i}}{\omega}, \quad i=1,2,} \\
\mathrm{e}^{u_{1}\left(l_{1}^{*}\right)}>\frac{\omega \overline{\left(a_{1}-h_{1}-D_{1}\right)}-\omega \overline{\left(c / m_{3}\right)}}{b_{1}^{M}}, \\
\mathrm{e}^{u_{2}\left(t_{2}^{*}\right)}>\frac{\omega \overline{\left(a_{2}-D_{2}\right)}}{b_{2}^{M}} .
\end{gathered}
$$

From (3.16), (3.17) and (3.20)-(3.22) we have

$$
\begin{aligned}
u_{i}(t) & <\ln \frac{\rho_{i}}{\omega}+\int_{0}^{\omega}\left|u_{i}^{\prime}(s)\right| \mathrm{d} s<\ln \frac{\rho_{i}}{\omega}+d_{i}, \quad i=1,2, \\
u_{1}(t) & >\ln \frac{\omega \overline{\left(a_{1}-h_{1}-D_{1}\right)}-\omega \overline{\left(c / m_{3}\right)}}{b_{1}^{M}}-\int_{0}^{\omega}\left|u_{1}^{\prime}(s)\right| \mathrm{d} s \\
& >\ln \frac{\omega \overline{\left(a_{1}-h_{1}-D_{1}\right)}-\omega \overline{\left(c / m_{3}\right)}}{b_{1}^{M}}-d_{1},
\end{aligned}
$$




$$
u_{2}(t)>\ln \frac{\omega \overline{\left(a_{2}-D_{2}\right)}}{b_{2}^{M}}-d_{2}
$$

So, for $t \in[0, \omega]$ we have

$$
\begin{gathered}
\left|u_{1}(t)\right|<\max \left\{\left|\ln \frac{\rho_{1}}{\omega}+d_{1}\right|,\left|\ln \frac{\omega \overline{\left(a_{1}-h_{1}-D_{1}\right)}-\omega \overline{\left(c / m_{3}\right)}}{b_{1}^{M}}-d_{1}\right|\right\} \stackrel{\text { def }}{=} R_{1}, \\
\left|u_{2}(t)\right|<\max \left\{\left|\ln \frac{\rho_{2}}{\omega}+d_{2}\right|,\left|\ln \frac{\omega \overline{\left(a_{2}-D_{2}\right)}}{b_{2}^{M}}-d_{2}\right|\right\} \stackrel{\text { def }}{=} R_{2} .
\end{gathered}
$$

From (3.5) we have

$$
\begin{aligned}
\mathrm{e}^{l} \int_{0}^{\omega} \mathrm{e}^{u_{3}(t)} \mathrm{d} t & <\int_{0}^{\omega} e(t) \mathrm{e}^{u_{3}(t)} \mathrm{d} t=\int_{0}^{\omega} \frac{f(t) \mathrm{e}^{u_{1}(t-\tau)}}{m_{1}(t)+m_{2}(t) \mathrm{e}^{u_{1}(t-\tau)}+m_{3}(t) \mathrm{e}^{u_{3}(t-\tau)}} \mathrm{d} t-\int_{0}^{\omega}\left[d(t)+h_{2}(t)\right] \mathrm{d} t \\
& <\omega \overline{\left(c / m_{2}\right)}-\omega \overline{\left(d+h_{2}\right)} .
\end{aligned}
$$

So, there exist $\eta_{1} \in(0, \omega)$ such that

$$
\mathrm{e}^{u_{3}\left(\eta_{1}\right)}<\frac{\overline{\left(f / m_{2}\right)}-\overline{\left(d+h_{2}\right)}}{\mathrm{e}^{l}} .
$$

From (3.5) we also have

$$
\int_{0}^{\omega} \frac{f(t) \mathrm{e}^{u_{1}(t-\tau)}}{m_{1}(t)+m_{2}(t) \mathrm{e}^{u_{1}(t-\tau)}+m_{3}(t) \mathrm{e}^{u_{3}(t-\tau)}} \mathrm{d} t<\omega \overline{\left(d+h_{2}\right)}+\mathrm{e}^{M} \int_{0}^{\omega} \mathrm{e}^{u_{3}(t)} \mathrm{d} t .
$$

It follows from (3.26) that there exist $\eta_{2} \in(0, \omega)$ such that

$$
\frac{f\left(\eta_{2}+\tau\right) \mathrm{e}^{u_{1}\left(\eta_{2}\right)}}{m_{1}\left(\eta_{2}+\tau\right)+m_{2}\left(\eta_{2}+\tau\right) \mathrm{e}^{u_{1}\left(\eta_{2}\right)}+m_{3}\left(\eta_{2}+\tau\right) \mathrm{e}^{u_{3}\left(\eta_{2}\right)}}<\omega \overline{\left(d+h_{2}\right)}+\mathrm{e}^{M} \mathrm{e}^{u_{3}\left(\eta_{2}+\tau\right)}
$$

So

$$
\mathrm{e}^{u_{3}\left(\eta_{2}\right)}>\frac{f\left(\eta_{2}+\tau\right) \mathrm{e}^{u_{1}\left(\eta_{2}\right)}}{\left\{\left(\overline{d+h_{2}}\right)+\frac{\mathrm{e}^{M}}{\mathrm{e}^{l}}\left[\overline{\left(\frac{f}{m_{2}}\right)}-\left(\overline{d+h_{2}}\right)\right]\right\} m_{3}\left(\eta_{2}+\tau\right)}-\frac{m_{1}\left(\eta_{2}+\tau\right)+m_{2}\left(\eta_{2}+\tau\right) \mathrm{e}^{u_{1}\left(\eta_{2}\right)} \text { def }}{m_{3}\left(\eta_{2}+\tau\right)}=p .
$$

It follows from (3.8), (3.25) and (3.27) that for $t \in[0, \omega]$ we have

$$
u_{3}(t)<\ln \frac{\overline{\left(f / m_{2}\right)}-\overline{\left(d+h_{2}\right)}}{\mathrm{e}^{l}}+d_{3}, \quad u_{3}(t)>\ln p-d_{3} .
$$

So $t \in[0, \omega]$ we have

$$
\left|u_{3}(t)\right|<\max \left\{\left|\ln \frac{\overline{\left(f / m_{2}\right)}-\overline{\left(d+h_{2}\right)}}{\mathrm{e}^{l}}+d_{3}\right|,\left|\ln p-d_{3}\right|\right\} \stackrel{\text { def }}{=} R_{3} .
$$

Clearly, $R_{i}(i=1,2,3)$ are independent of $\lambda$. On other hand, we consider the following algebraic equation 


$$
\left\{\begin{array}{l}
\left(\overline{a_{1}-h_{1}-D_{1}}\right)-\bar{b}_{1} \mathrm{e}^{u_{1}}-\frac{c \mathrm{e}^{u_{3}}}{\bar{m}_{1}+\bar{m}_{2} \mathrm{e}^{u_{1}}+\bar{m}_{3} \mathrm{e}^{u_{3}}}+\bar{D}_{1} \mathrm{e}^{u_{2}-u_{1}}=0 \\
\left(\overline{a_{2}-D_{2}}\right)-\bar{b}_{2} \mathrm{e}^{u_{2}}+\bar{D}_{2} \mathrm{e}^{u_{1}-u_{2}}=0 \\
\left(\overline{-d-h_{2}}\right)-\bar{e} \mathrm{e}^{u_{3}}+\frac{\bar{f}^{u_{1}}}{\bar{m}_{1}+\bar{m}_{2} \mathrm{e}^{u_{1}}+\bar{m}_{3} \mathrm{e}^{u_{3}}}=0
\end{array}\right.
$$

Take $\rho=R_{1}+R_{2}+R_{3}+R_{0}$, where $R_{0}$ is large enough such that the solution $u^{*}=\left(u_{1}^{*}, u_{2}^{*}, u_{3}^{*}\right)^{\mathrm{T}}$ of (3.28) satisfies

$$
\left\|u^{*}\right\|=\left\|\left(u_{1}^{*}, u_{2}^{*}, u_{3}^{*}\right)^{\mathrm{T}}\right\|<\rho .
$$

Let $\Omega=\{u \in X:\|u\|<\rho\}$, then $\Omega$ satisfies the condition (1) in Lemma 2.1. When $u \in \partial \Omega \cap K e r L=\partial \Omega \cap R^{3}$, $u$ is a constant vector in $R^{3}$ and $\|u\|=\rho$. It follows from the definition of $\rho$ that $Q N(u) \neq 0$, so the condition (2) in Lemma 2.1 is satisfied. In order to verify the condition (3) in Lemma 2.1, we define $\phi: D o m L \times[0,1] \rightarrow X$ by

$$
\phi\left(u_{1}, u_{2}, u_{3}, \mu\right)=\left(\begin{array}{c}
\left(\overline{a_{1}-h_{1}-D_{1}}\right)-\bar{b}_{1} \mathrm{e}^{u_{1}(t)} \\
\left(\overline{a_{2}-D_{2}}\right)-\bar{b}_{2} \mathrm{e}^{u_{2}(t)} \\
\bar{f} \mathrm{e}^{u_{1}(t)} \\
\left(\overline{-d-h_{2}}\right)+\frac{\bar{c}^{u_{3}(t)}}{\bar{m}_{1}+\bar{m}_{2} \mathrm{e}^{u_{1}(t)}+\bar{m}_{3} \mathrm{e}^{u_{3}(t)}}
\end{array}\right)+\mu\left(\begin{array}{c}
\frac{\bar{m}_{1}+\bar{m}_{2} \mathrm{e}^{u_{1}(t)}+\bar{m}_{3} \mathrm{e}^{u_{3}(t)}+\bar{D}_{1} \mathrm{e}^{u_{2}(t)-u_{1}(t)}}{\bar{D}_{2} \mathrm{e}^{u_{1}(t)-u_{2}(t)}} \\
-\bar{e}^{u_{3}(t)}
\end{array}\right),
$$

where $u_{i} \in R,(i=1,2,3), \mu \in[0,1]$ is a parameter. When $u \in \partial \Omega \cap \operatorname{Ker} L=\partial \Omega \bigcap R^{3}, u$ is a constant vector in $R^{3}$ and $\|u\|=\rho$. It is easy to obtain that $u \in \partial \Omega \cap \operatorname{Ker} L$, then $\phi\left(u_{1}, u_{2}, u_{3}, \mu\right) \neq 0$. So, $\phi\left(u_{1}, u_{2}, u_{3}, \mu\right)$ is a Homotopy mapping, due to homogoy invariance theorem of topology degree, we have

$$
\begin{aligned}
& \operatorname{deg}\left\{Q N(u), \Omega \cap \operatorname{KerL},(0,0,0)^{\mathrm{T}}\right\} \\
& =\operatorname{deg}\left\{\phi\left(u_{1}, u_{2}, u_{3}, 1\right), \Omega \cap \operatorname{KerL},(0,0,0)^{\mathrm{T}}\right\} \\
& =\operatorname{deg}\left\{\phi\left(u_{1}, u_{2}, u_{3}, 0\right), \Omega \cap \operatorname{KerL},(0,0,0)^{\mathrm{T}}\right\} \\
& =\operatorname{deg}\left\{\left(\overline{a_{1}-h_{1}-D_{1}}\right)-\bar{b}_{1} \mathrm{e}^{u_{1}(t)},\left(\overline{a_{2}-D_{2}}\right)-\bar{b}_{2} \mathrm{e}^{u_{2}(t)},\left(\overline{-d-h_{2}}\right)+\frac{\overline{\mathrm{f}^{u_{1}(t)}}}{\left.\bar{m}_{1}+\bar{m}_{2} \mathrm{e}^{u_{1}(t)}+\bar{m}_{3} \mathrm{e}^{u_{3}(t)}, \Omega \cap \operatorname{KerL},(0,0,0)^{\mathrm{T}}\right\} .}\right.
\end{aligned}
$$

It is not difficult to see that the following algebraic equation

$$
\left\{\begin{array}{l}
\left(\overline{a_{1}-h_{1}-D_{1}}\right)-\bar{b}_{1} \mathrm{e}^{u_{1}}=0 \\
\left(\overline{a_{2}-D_{2}}\right)-\bar{b}_{2} \mathrm{e}^{u_{2}}=0 \\
\left(\overline{-d-h_{2}}\right)+\frac{\bar{f} \mathrm{e}^{u_{1}}}{\bar{m}_{1}+\bar{m}_{2} \mathrm{e}^{u_{1}}+\bar{m}_{3} \mathrm{e}^{u_{3}}}=0
\end{array}\right.
$$

has a unique solution

$$
y_{1}^{*}=\frac{\left(\overline{a_{1}-h_{1}-D_{1}}\right)}{\bar{b}_{1}}, \quad y_{2}^{*}=\frac{\left(\overline{a_{2}-D_{2}}\right)}{\bar{b}_{2}}, \quad y_{3}^{*}=\frac{\bar{f} y_{1}^{*}-\left(\bar{m}_{1}+\bar{m}_{2} y_{1}^{*}\right)\left(\overline{d+h_{2}}\right)}{\left(\overline{d+h_{2}}\right) \bar{m}_{3}} .
$$

Thus 


$$
\begin{aligned}
& \operatorname{deg}\left\{Q N(u), \Omega \cap \operatorname{KerL},(0,0,0)^{\mathrm{T}}\right\} \\
& =\left|\begin{array}{ccc}
-\bar{b}_{1} & 0 & 0 \\
\frac{0}{f m_{1}}+\overline{f m_{3}} y_{3}^{*} & -\bar{b}_{2} & 0 \\
\left.\hline \bar{m}_{1}+\bar{m}_{2} y_{1}^{*}+\bar{m}_{3} y_{3}^{*}\right)^{2} & 0 & \frac{-\overline{f m_{3}} y_{1}^{*}}{\left(\bar{m}_{1}+\bar{m}_{2} y_{1}^{*}+\bar{m}_{3} y_{3}^{*}\right)^{2}}
\end{array}\right|=-1 \neq 0 .
\end{aligned}
$$

By now we have proved the condition (3) in Lemma 2.1. This completes the proof of Theorem 3.1.

\section{References}

[1] Freedman, H.I. (1980) Mathematical Models in Population Ecology. Marcel Dekker, New York.

[2] Sugie, J. (1998) Two-Parameter Bifurcation System of Ivlev Type. Journal of Mathematical Analysis and Applications, 217, 349-371. http://dx.doi.org/10.1006/jmaa.1997.5700

[3] Ardito, A. and Ricciardi, P. (1995) Lyapunov Functions for a Generalized Gause-Type Model. Journal of Mathematical Biology, 33, 816-828. http://dx.doi.org/10.1007/BF00187283

[4] Hassel, M.P. (1978) The Dynamics of Arthropod Predator-Prey Systems. Princeton University Press, Princeton.

[5] Hwang, T.W. (1999) Predator-Prey System. Journal of Mathematical Analysis and Applications, 238, 179-195. http://dx.doi.org/10.1006/jmaa.1999.6520

[6] Hsu, S.B., Hwang, T.W. and Kuang, Y. (2001) Global Analysis of the Michaelis-Menten Type Ratio-Dependent Predator-Prey. Journal of Mathematical Biology, 42, 489-506. http://dx.doi.org/10.1007/s002850100079

[7] Kot, M. (2001) Elements of Mathematical Biology. Cambridge University Press, Cambridge.

[8] Kuang, Y. and Freedman, H.I. (1988) Uniqueness of Limit Cycles in Gause-Type Predator-Prey Systems. Mathematical Biosciences, 88, 67-84. http://dx.doi.org/10.1016/0025-5564(88)90049-1

[9] Kooij, R.E. and Zegeling, A. (1996) A Predator-Prey Model with Ivlev’s Functional Response. Journal of Mathematical Analysis and Applications, 198, 473-489. http://dx.doi.org/10.1006/jmaa.1996.0093

[10] Liu, X.X. and Lou, Y.J. (2010) Global Dynamics of a Predator-Prey Model. Journal of Mathematical Analysis and Applications, 371, 323-340. http://dx.doi.org/10.1016/j.jmaa.2010.05.037

[11] Xiao, D.M., Li, W.X. and Han, M.A. (2006) Dynamics in Ratio-Dependent Predator-Prey Model with Predator Harvesting. Journal of Mathematical Analysis and Applications, 324, 14-29. http://dx.doi.org/10.1016/j.jmaa.2005.11.048

[12] Xiao, D. and Zhang, Z.D. (2003) On the Uniqueness and Nonexistence of Limit Cycles for Predator-Prey Systems. Nonlinearity, 16, 1185-1201. http://dx.doi.org/10.1088/0951-7715/16/3/321

[13] Beddington, J.R. (1975) Mutual Interference between Parasites or Predators and Its Effect on Searching Efficiency. Journal of Animal Ecology, 3, 331-340. http://dx.doi.org/10.2307/3866

[14] DeAngelis, D.L., Goldstein, R.A. and O’Neil, R.V. (1975) A Model for Trophic Interaction. Ecology, 4, 881-892. http://dx.doi.org/10.2307/1936298

[15] Cantrell, R.S. and Cosner, C. (2001) On the Dynamics of Predator-Prey Models with the Beddington-DeAngelis Functional Response. Journal of Mathematical Analysis and Applications, 257, 206-222. http://dx.doi.org/10.1006/jmaa.2000.7343

[16] Chen, F., Chen, Y. and Shi, J. (2008) Stability of the Boundary Solution of a Nonautonomous Predator-Prey System with the Beddington-DeAngelis Functional Response. Journal of Mathematical Analysis and Applications, 344, 10571067. http://dx.doi.org/10.1016/j.jmaa.2008.03.050

[17] Cui, J. and Takeuchi, Y. (2006) Permanence, Extinction and Periodic Solution of Predator-Prey System with the Beddington-DeAngelis Functional Response. Journal of Mathematical Analysis and Applications, 317, 464-474. http://dx.doi.org/10.1016/j.jmaa.2005.10.011

[18] Dimitrov, D.T. and Kojouharov, H.V. (2005) Complete Mathematical Analysis of Predator-Prey System with Linear Prey Growth and Beddington-DeAngelis Functional Response. Applied Mathematics and Computation, 162, 523-538. http://dx.doi.org/10.1016/j.amc.2003.12.106

[19] Fan, M. and Kuang, Y. (2004) Dynamics of a Nonautonomous Predator-Prey System with the Beddington-DeAngelis Functional Response. Journal of Mathematical Analysis and Applications, 295, 15-39. http://dx.doi.org/10.1016/j.jmaa.2004.02.038 
[20] Hwang, T.W. (2003) Global Analysis of the Predator-Prey System with the Beddington-DeAngelis Functional Response. Journal of Mathematical Analysis and Applications, 281, 395-401.

http://dx.doi.org/10.1016/S0022-247X(02)00395-5

[21] Liu, S. and Beretta, E. (2006) A Stage-Structured Predator-Prey Model of Beddington-DeAngelis Type. SIAM Journal on Applied Mathematics, 66, 1101-1129. http://dx.doi.org/10.1137/050630003

[22] Li, H.Y. and Takeuchi, Y. (2011) Dynamics of the Density Dependent Predator-Prey System with Beddington-DeAngelis Functional Response. Journal of Mathematical Analysis and Applications, 374, 644-654. http://dx.doi.org/10.1016/j.jmaa.2010.08.029

[23] Gaines, R.E. and Mawhin, J.L. (1977) Coincidence Degree and Nonlinear Differential Equations. Springer, Berlin. 